\title{
A GENERALIZATION OF HERMITE'S INTERPOLATION FORMULA IN TWO VARIABLES
}

\author{
M. M. CHAWLA and N. JAYARAJAN
}

(Received 17 July 1972)

Communicated by E. Strzelecki

\section{Introduction}

Spitzbart [1] has considered a generalization of Hermite's interpolation formula in one variable and has obtained a polynomial $p(x)$ of degree $n+\sum_{j=0}^{n} r_{j}$ in $x$ which interpolates to the values of a function and its derivatives up to order $r_{j}$ at $x_{j}, j=0,1, \cdots, n$. Ahlin [2] has considered a bivariate generalization of Hermite's interpolation formula. He has developed a bivariate osculatory interpolation polynomial which agrees with $f(x, y)$ and its partial and mixed partial derivatives up to a specified order at each of the nodes of a Cartesian grid. However, the above interpolation problem considered by Ahlin assumes that the values of partial and mixed partial derivatives of the same fixed order $k-1$ are available at every point of the rectangular grid. It may also be observed that Ahlin's formula is essentially a Cartesian product of a special case of Spitzbart's formula in one variable.

In the present paper, we consider a bivariate generalization of Spitzbart's formula. We discuss the bivariate interpolation problem in which at any point $\left(x_{i}, y_{j}\right), i=0,1, \cdots, m$ and $j=0,1, \cdots, n$ of a Cartesian grid, the maximum order of the partial derivative with respect to $x$ depends only on $i$ and the maximum order of the partial derivative with respect to $y$ depends only on $j$. In other words, we consider interpolation to the data

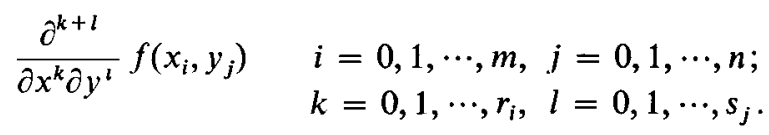

The resulting interpolation formula might also be applicable in a situation where only the function values might be available but no partial derivatives with respect to $x$ along some $x=x_{i}$ or no partial derivatives with respect to $y$ along some $y=y_{j}$. Ahlin's interpolation formula becomes a particular case of our formula when $r_{i}=s_{j}=k-1$ (fixed) for all $i$ and $j$. 


\section{The interpolation formula}

We first state the generalized Hermite's interpolation formula considered by Spitzbart [1].

THEOREM 1. Let there be given $x_{i}, r_{i}, f_{i}^{(k)}, i=0,1, \cdots, m ; k=0,1, \cdots, r_{i}$. Let $p_{i}(x)$ and $g_{i}(x)$ be defined by

(1) $p_{i}(x)=\left(x-x_{0}\right)^{r_{0}+1} \cdots\left(x-x_{i-1}\right)^{r_{i-1}+1}\left(x-x_{i+1}\right)^{r_{i+1}+1} \cdots\left(x-x_{m}\right)^{r_{m}+1}$

(2) $g_{i}(x)=\left[p_{i}(x)\right]^{-1}$.

Then the unique polynomial $H_{M}(x)$ of degree $M=m+\sum_{i=0}^{m} r_{i}$ such that

$$
H_{M}^{(k)}\left(x_{i}\right)=f_{i}^{(k)}, \quad i=0,1, \cdots, m ; k=0,1, \cdots, r_{i},
$$

(here, $\left.H^{(k)}(x)=\left(d^{k} / d x^{k}\right) H(x)\right)$ is given by

$$
H_{M}(x)=\sum_{i=0}^{m} \sum_{k=0}^{r_{i}} A_{l k}(x) f_{i}^{(k)}
$$

where

$$
A_{i k}(x)=p_{i}(x) \frac{\left(x-x_{i}\right)^{k}}{k !} \sum_{t=0}^{r_{i}-k} \frac{1}{t !} g_{i}^{(t)}\left(x_{i}\right)\left(x-x_{i}\right)^{t} .
$$

The fundamental polynomials $A_{i k}(x)$ satisfy

$$
\begin{aligned}
& A_{i_{1} k}^{(u)}\left(x_{i}\right)=\delta_{i_{1}} \delta_{u k}, \quad i_{1}, i=0,1, \cdots, m ; \\
& k=0,1, \cdots, r_{i_{1}}, u=0,1, \cdots, r_{i},
\end{aligned}
$$

where $\delta_{i r}$ is the Kronecker delta function.

Our main result is the following bivariate generalization of Theorem 1.

THEOREM 2. Let there be given a set of values

$$
\begin{aligned}
& f_{i, j}^{(k, l)}, \quad i=0,1, \cdots, m, j=0,1, \cdots, n ; \\
& k=0,1, \cdots, r_{i}, \quad l=0,1, \cdots, s_{j} .
\end{aligned}
$$

Then the unique polynomial $H_{M, N}(x, y)$ of degree $M=m+\sum_{i=0}^{m} r_{i}$ in $x$ and of degree $N=n+\sum_{j=0}^{n} s_{j}$ in $y$ such that

$$
\frac{\partial^{k+l}}{\partial x^{k} \partial y^{l}} H_{M, N}\left(x_{i}, y_{j}\right)=f_{i, j}^{(k, l)}, \quad \begin{aligned}
i & =0,1, \cdots, m, j=0,1, \cdots, n ; \\
k & =0,1, \cdots, r_{i}, l=0,1, \cdots, s_{j}
\end{aligned}
$$

is given by 


$$
H_{M, N}(x, y)=\sum_{i=0}^{m} \sum_{j=0}^{n} \sum_{k=0}^{r_{i}} \sum_{l=0}^{s_{j}} A_{i k}(x) B_{j l}(y) f_{i, j}^{(k, l)}
$$

where $A_{i k}(x)$ are the same as in (5), and

$$
B_{j l}(y)=q_{j}(y) \frac{\left(y-y_{j}\right)^{l}}{l !} \sum_{t=0}^{s_{j}-1} \frac{h_{j}^{(t)}\left(y_{j}\right)}{t !}\left(y-y_{j}\right)^{t}
$$

where

$$
q_{j}(y)=\left(y-y_{0}\right)^{s_{0}+1} \cdots\left(y-y_{j-1}\right)^{s_{j-1}+1}\left(y-y_{j+1}\right)^{s_{j+1}+1} \cdots\left(y-y_{n}\right)^{s_{n}+1}
$$

and

$$
h_{j}(y)=\left[q_{j}(y)\right]^{-1} .
$$

Proof. We first observe that the total number of the given data $f_{i, j}^{(k, l)}$ is $\sum_{i=0}^{m} \sum_{j=0}^{n}\left(r_{i}+1\right)\left(s_{j}+1\right)$ which is equal to the number of (unknown) coefficients in a polynomial of maximum degree $N$ in $x$ and $N$ in $y$.

Now, the polynomials $A_{i k}(x)$ in (8) are the same as the fundamental polynomials of Theorem 1 and satisfy (6), therefore, the polynomials $B_{j l}(y)$ which have been defined in an analogous manner satisfy

$$
\begin{aligned}
& B_{j_{1}}^{(v)}\left(y_{j}\right)=\delta_{j_{1}} \delta_{v l}, \quad j_{1}, j=0,1, \cdots, n ; \\
& l=0,1, \cdots, s_{j_{1}}, v=0,1, \cdots, s_{j} .
\end{aligned}
$$

We next verify that the polynomial $H_{M, N}(x, y)$ defined in (8) satisfies the interpolation conditions (7). Since

$$
\frac{\partial^{u+v}}{\partial x^{u} \partial y^{v}} H_{M, N}\left(x_{i}, y_{j}\right)=\sum_{i_{1}=0}^{m} \sum_{j_{1}=0}^{n} \sum_{k=0}^{r_{i 1}} \sum_{l=0}^{s_{j 1}} A_{i_{1} k}^{(u)}\left(x_{i}\right) B_{j_{1} l}^{(v)}\left(y_{j}\right) f_{i_{1}, j_{1}}^{(k, l)}
$$

using (6) and (12) it follows that

$$
\begin{aligned}
\frac{\partial^{u+v}}{\partial x^{u} \partial y^{v}} H_{M, N}\left(x_{i}, y_{j}\right) & =\sum_{i_{1}=0}^{m} \sum_{j,=0}^{n} \sum_{k=0}^{r_{i 1}} \sum_{l=0}^{s_{j}} \delta_{i_{1} i} \delta_{u k} \delta_{j_{1} j} \delta_{v l} f_{i_{1}, j_{1}}^{(k, l)} \\
& =f_{i, j}^{(u, v)}, \quad \begin{aligned}
i & =0,1, \cdots, m, j=0,1, \cdots, n ; \\
u & =0,1, \cdots, r_{i}, v=0,1, \cdots, s_{j} .
\end{aligned}
\end{aligned}
$$

For the uniqueness of $H_{M N}(x, y)$, suppose there exists another polynomial $H_{M N}^{*}(x, y)$ of maximum degree $M$ in $x$ and of maximum degree $N$ in $y$ which also satisfies the interpolation conditions (7). Then,

$$
T(x, y)=H_{M, N}^{*}(x, y)-H_{M, N}(x, y)
$$

is a polynomial of maximum degree $M$ in $x$ and of maximum degree $N$ in $y$ such that 


$$
\frac{\partial^{k+l}}{\partial x^{k} \partial y^{l}} T\left(x_{i}, y_{j}\right)=0, \quad \begin{aligned}
& i=0,1, \cdots, m, j=0,1, \cdots, n \\
& k=0,1, \cdots, r_{i}, l=0,1, \cdots, s_{j} .
\end{aligned}
$$

Along any one of the mesh lines $y=y_{j}, T\left(x, y_{j}\right)$ is a polynomial of maximum degree $M$ in $x$ such that

$$
T\left(x_{i}, y_{j}\right)=\frac{\partial}{\partial x} T\left(x_{i}, y_{j}\right)=\cdots=\frac{\partial^{r_{i}}}{\partial x^{r_{i}}} T\left(x_{i}, y_{j}\right)=0 .
$$

Hence, $\left(x-x_{i}\right)^{r_{i}+1}$ must be a factor of $T\left(x, y_{j}\right)$ for $i=0,1, \cdots, m$. Thus,

$$
T\left(x, y_{j}\right)=K\left(x-x_{0}\right)^{r_{0}+1} \cdots\left(x-x_{m}\right)^{r_{m}+1}
$$

where $K$ is a constant. Since the right side is a polynomial of degree $M+1$ in $x$, and $T\left(x, y_{j}\right)$ is of maximum degree $M$ in $x$, comparing the coefficient of $x^{N+1}$ on either side, it follows that $K=0$. Hence,

$$
T\left(x, y_{j}\right) \equiv 0, \quad j=0,1, \cdots, n .
$$

By a similar reasoning we obtain

$$
\frac{\partial^{l}}{\partial y^{l}} T\left(x, y_{j}\right) \equiv 0, \quad l=1,2, \cdots, s_{j} ; j=0,1, \cdots, n .
$$

Now choose any arbitrary line $x=\xi$. Using (14) and (15) it follows after a similar argument that

$$
T(\xi, y) \equiv 0
$$

Since the choice of $\xi$ is arbitrary, we conclude that $T(x, y) \equiv 0$, proving the uniqueness of $H_{M, N}(x, y)$. This completes the proof of Theorem 2.

The Taylor two-point interpolation formula (Davis [3], page 37) is a particular case of Theorem 1.

Corollary 1. The unique polynomial $H_{2 n-1}(x)$ in $x$ of degree $2 n-1$ which interpolates to the data $f_{a}^{(k)}, f_{b}^{(k)}, k=0,1, \cdots, n-1$, is given $b y$

$$
H_{2 n-1}(x)=(x-b)^{n} \sum_{k=0}^{n-1} \frac{A_{k}}{k !}(x-a)^{k}+(x-a)^{n} \sum_{k=0}^{n-1} \frac{B_{k}}{k !}(x-b)^{k}
$$

where

and

$$
A_{k}=\left[\frac{d^{k}}{d x^{k}} \frac{f(x)}{(x-b)^{n}}\right]_{x=a}
$$

$$
B_{k}=\left[\frac{d^{k}}{d x^{k}} \frac{f(x)}{(x-a)^{n}}\right]_{x=b}
$$

We note that, from Theorem 1 , 


$$
\begin{aligned}
H_{2 n-1}(x) & =(x-b)^{n} \sum_{k=0}^{n-1} \frac{(x-a)^{k}}{k !} \sum_{t=0}^{n-1-k} \frac{\left\{D^{t}(x-b)^{-n}\right\}_{x=a}}{t !}(x-a)^{t} f_{a}^{(k)} \\
& +(x-a)^{n} \sum_{k=0}^{n-1} \frac{(x-b)^{k}}{k !} \sum_{t=0}^{n-1-k} \frac{\left\{D^{t}(x-a)^{-n}\right\}_{x=b}}{t !}(x-b)^{t} f_{b}^{(k)}
\end{aligned}
$$

where $D \equiv d / d x$. Simplifying the summations in the two terms of the right side of (20) and with the help of Leibnitz theorem, we get

$$
H_{2 n-1}(x)=(x-b)^{n} \sum_{k=0}^{n-1} \frac{A_{k}}{k !}(x-a)^{k}+(x-a)^{n} \sum_{k=0}^{n-1} \frac{B_{k}}{k !}(x-b)^{k},
$$

where $A_{k}$ and $B_{k}$ are given by (18) and (19).

The following result is a particular case of our Theorem 2, and may be regarded as a two-dimensional generalization of the above two-point Taylor interpolation formula.

COROLlary, 2. The unique polynomial $H_{2 n-1,2 n-1}(x, y)$ of degree $2 n-1$ in $x$ and of degree $2 n-1$ in $y$ which satisfies the interpolation conditions:

$$
\frac{\partial^{k+l}}{\partial x^{k} \partial y^{l}} H_{2 n-1,2 n-1}\left(x_{i}, y_{j}\right)=f_{i, j}^{(k, l)}, \quad \begin{aligned}
& i=0,1, j=0,1 ; \\
& k, l=0,1, \cdots, n-1 ;
\end{aligned}
$$

$x_{0}=a, x_{1}=b ; y_{0}=c, y_{1}=d$, is given $b y$

$$
\begin{aligned}
H_{2 n-1,2 n-1}(x, y) & =(x-a)^{n}(y-c)^{n} \sum_{k=0}^{n-1} \sum_{l=0}^{n-1} A_{k l} \frac{(x-b)^{k}}{k !} \frac{(y-d)^{l}}{l !} \\
& +(x-a)^{n}(y-d)^{n} \sum_{k=0}^{n-1} \sum_{l=0}^{n-1} B_{k l} \frac{(x-b)^{k}}{k !} \frac{(y-c)^{l}}{l !} \\
& +(x-b)^{n}(y-c)^{n} \sum_{k=0}^{n-1} \sum_{l=0}^{n-1} C_{k l} \frac{(x-a)^{k}}{k !} \frac{(y-d)^{l}}{l !} \\
& +(x-b)^{n}(y-d)^{n} \sum_{k=0}^{n-1} \sum_{l=0}^{n-1} D_{k l} \frac{(x-a)^{k}}{k !} \frac{(y-c)^{l}}{l !},
\end{aligned}
$$

where

$$
A_{k l}=\left[\frac{\partial^{k+l}}{\partial x^{k} \partial y^{l}} \frac{f(x, y)}{(x-a)^{n}(y-c)^{n}}\right]_{x=b, y=d}
$$

with similar expressions for $B_{k l}, C_{k l}$, and $D_{k l}$.

The above result can be established from Theorem 2 by proceeding the same way as in Corollary 1. 


\section{Illustration}

Suppose that the values of a function $f(x, y)$ as well as the values of $\partial f / \partial x$ are known at the four corners $( \pm 1, \pm 1)$ of a square. Then the unique polynomial of degree 3 in $x$ and of degree 1 in $y$ which interpolates to these values is given by

$$
\begin{aligned}
H_{3,1}(x, y)= & (1 / 8)\left[(x-1)^{2}(x+2)(1-y) f(-1,-1)\right. \\
& +(x-1)^{2}(x+2)(y+1) f(-1,1)+(x+1)^{2}(2-x)(1-y) f(1,-1) \\
& +(x+1)^{2}(2-x)(y+1) f(1,1) \\
& +(x-1)^{2}(x+1)(1-y) f_{x}(-1,-1) \\
& +(x-1)^{2}(x+1)(y+1) f_{x}(-1,1) \\
& \left.+(x+1)^{2}(x-1)(1-y) f_{x}(1,-1)+(x+1)^{2}(x-1)(y+1) f_{x}(1,1)\right]
\end{aligned}
$$

where $f_{x}=\partial f / \partial x$.

For the particular function $f(x, y)=1 /\left(16+x^{2}+y\right)$, the interpolation polynomial $H_{3,1}(x, y)$ becomes

$$
\begin{aligned}
H_{3,1}(x, y)=(1 / 8) & {\left[(1 / 16)(x-1)^{2}(x+2)(1-y)+(1 / 18)(x-1)^{2}(x+2)(y+1)\right.} \\
& +(1 / 16)(x+1)^{2}(2-x)(1-y)+(1 / 18)(x+1)^{2}(2-x)(1+y) \\
& +(1 / 128)(x-1)^{2}(x+1)(1-y) \\
& +(1 / 162)(x-1)^{2}(x+1)(y+1) \\
& -(1 / 128)(x+1)^{2}(x-1)(1-y) \\
& \left.-(1 / 162)(x+1)^{2}(x-1)(y+1)\right] .
\end{aligned}
$$

Computing the values at the origin, we obtain $f(0,0)=1 / 16$, while $H_{3.1}(0,0)$ $=2593 / 41472$. Thus, the approximation $f(0,0) \approx H_{3,1}(0,0)$ has an error of $1 / 41472$ !

Next, consider the function $f(x, y)=1 /\left(16+x^{2}+\sqrt{ } y\right)$ over $0 \leqq x \leqq 1$, $0 \leqq y \leqq 1$. The partial derivatives of $f$ with respect to $y$ do not exist along $y=0$. However, from Theorem 2 we can obtain an osculatory interpolation polynomial interpolating to the values of $f$ at $(0,0),(0,1),(1,0),(1,1)$, to the values of the partial derivatives with respect to $x$ (only) at $(0,0)$ and $(1,0)$, and to the values of the partial and mixed partial derivatives up to any order at the points $(0,1)$ and $(1,1)$.

This example distinguishes our interpolation formula (Theorem 2) from that of Ahlin [2].

\section{The error of interpolation}

We next derive expressions for the error of the interpolation formula given in Theorem 2 for the following two classes of functions:

$$
\frac{\partial^{M+N+2}}{\partial x^{M+1} \partial y^{N+1}} f(x, y) \text { is continuous, }
$$


(ii) $f(x, y)$ can be continued analytically as a single valued and regular function $f(z, w)$ of two complex variables in a certain cross-product region $D_{z} \times D_{w}$.

\section{Error in terms of partial derivatives}

The error of the interpolation formula of Theorem 1 is given (Davis [3], page 67) by

$$
\begin{aligned}
R(x) & =f(x)-H_{M}(x) \\
& =\left(x-x_{0}\right)^{r_{0}+1} \cdots\left(x-x_{m}\right)^{r_{m}+1} \frac{f^{(M+1)}(\xi)}{(M+1) !}
\end{aligned}
$$

where $\min \left(x ; x_{0}, \cdots, x_{m}\right) \leqq \xi \leqq \max \left(x ; x_{0}, \cdots, x_{m}\right)$; that is,

$$
f(x)=H_{M}(x)+R(x) .
$$

In the case of two variables, if we keep $y$ fixed, we can write

$$
f(x, y)=\sum_{i=0}^{m} \sum_{k=0}^{r_{i}} A_{i k}(x) \frac{\partial^{k}}{\partial x^{k}} f\left(x_{i}, y\right)+\frac{\alpha(x)}{(M+1) !} \frac{\partial^{M+1}}{\partial x^{M+1}} f(\xi, y)
$$

where

$$
\alpha(x)=\left(x-x_{0}\right)^{r_{0}+1} \cdots\left(x-x_{m}\right)^{r_{m}+1}
$$

and $\min \left(x ; x_{0}, \cdots, x_{m}\right) \leqq \xi \leqq \max \left(x ; x_{0}, \cdots, x_{m}\right)$. Similarly, if $x$ is kept fixed,

$$
f(x, y)=\sum_{j=0}^{n} \sum_{l=0}^{s_{j}} B_{j l}(y) \frac{\partial^{l}}{\partial y^{l}} f\left(x, y_{j}\right)+\frac{\beta(y)}{(N+1) !} \frac{\partial^{N+1}}{\partial y^{N+1}} f(x, \eta)
$$

where

$$
\beta(y)=\left(y-y_{0}\right)^{s_{0}+1} \cdots\left(y-y_{n}\right)^{s_{n}+1}
$$

and $\min \left(y ; y_{0}, \cdots, y_{n}\right) \leqq \eta \leqq \max \left(y ; y_{0}, \cdots, y_{n}\right)$. From (25) and (27), it follows that

$$
\begin{aligned}
f(x, y)= & \sum_{i=0}^{m} \sum_{j=0}^{n} \sum_{k=0}^{r_{l}} \sum_{l=0}^{s_{j}} A_{t k}(x) B_{j l}(y) \frac{\partial^{k+l}}{\partial x^{k} \partial y^{l}} f\left(x_{i}, y_{j}\right)+ \\
& \frac{\alpha(x)}{(M+1) !} \sum_{j=0}^{n} \sum_{l=0}^{s_{j}} B_{j l}(y) \frac{\partial^{M+1+l}}{\partial x^{M+1} \partial y^{l}} f\left(\xi, y_{j}\right) \\
& +\frac{\beta(y)}{(N+1) !} \sum_{i=0}^{m} \sum_{k=0}^{r_{i}} A_{t k}(x) \frac{\partial^{k+N+1}}{\partial x^{k} \partial y^{N+1}} f\left(x_{i}, \eta\right) \\
& +\frac{\alpha(x) \beta(y)}{(M+1) !(N+1) !} \frac{\partial^{M+N+2}}{\partial x^{M+1} \partial y^{N+1}} f(\xi, \eta)
\end{aligned}
$$


that is,

$$
f(x, y)=H_{M, N}(x, y)+R(x, y) \text {. }
$$

Now, from (25),

(30)

$\sum_{i=0}^{m} \sum_{k=0}^{r_{i}} A_{i k}(x) \frac{\partial^{k+N+1}}{\partial x^{k} \partial y^{N+1}} f\left(x_{i}, \eta\right)=\frac{\partial^{N+1}}{\partial y^{N+1}}\left[f(x, y)-\frac{\alpha(x)}{(M+1) !} \frac{\partial^{M+1}}{\partial x^{M+1}} f(\xi, y)\right]_{y=\eta}$ and, from (27),

$$
\sum_{j=0}^{n} \sum_{l=0}^{s_{j}} B_{j l}(y) \frac{\partial^{M+1+l}}{\partial x^{M+1} \partial y^{l}} f\left(\xi, y_{j}\right)=\frac{\partial^{M+1}}{\partial x^{M+1}}\left[f(x, y)-\frac{\beta(y)}{(N+1) !} \frac{\partial^{N+1}}{\partial y^{N+1}} f(x, \eta)\right]_{x=\xi} .
$$

Substituting (31) and (30) into the second and third terms, respectively, of the right side of (29), we obtain

$$
\begin{aligned}
R(x, y)= & \frac{\alpha(x)}{(M+1) !} \frac{\partial^{M+1}}{\partial x^{M+1}} f(\xi, y)+\frac{\beta(y)}{(N+1) !} \frac{\partial^{N+1}}{\partial y^{N+1}} f(x, \eta) \\
& -\frac{\alpha(x) \beta(y)}{(M+1) !(N+1) !} \frac{\partial^{M+N+2}}{\partial x^{M+1} \partial y^{N+1}} f(\xi, \eta),
\end{aligned}
$$

which gives the error of interpolation.

\section{Error in terms of contour integrals}

In the case of a single variable $x$, let $C$ be a closed contour in the region $D_{z}$ of analytic continuation of $f(x)$ containing the points $x_{0}, \cdots, x_{m}$ in its interior. By applying the residue theorem to the contour integral

$$
\frac{1}{2 \pi i} \int_{C} \frac{f(z) d z}{\alpha(z)(z-x)}
$$

with $\alpha(z)$ defined in (26), we obtain

$$
f(x)=H_{M}(x)+\frac{\alpha(x)}{2 \pi i} \int_{C} \frac{f(z) d z}{\alpha(z)(z-x)}
$$

where $H_{M}(x)$ is the interpolation polynomial of Theorem 1 .

Applying (33) to a function $f(x, y)$ of two variables, and keeping $y$ fixed, we obtain

$$
f(x, y)=\sum_{i=0}^{m} \sum_{k=0}^{r_{i}} A_{i k}(x) \frac{\partial^{k}}{\partial x^{k}} f\left(x_{i}, y\right)+\frac{\chi(x)}{2 \pi i} \int_{C_{1}} \frac{f(z, y) d z}{\alpha(z)(z-x)}
$$

where $C_{1}$ is a simple closed contour in the region $D_{z}$ of analyticity of $f(z, y)$ ( $y$ fixed) and containing the points $x_{0}, \cdots, x_{m}$ in its interior. Similarly, if $x$ is 
fixed, we can write

$$
f(x, y)=\sum_{j=0}^{n} \sum_{l=0}^{s_{j}} B_{j l}(y) \frac{\partial^{l}}{\partial y^{l}} f\left(x, y_{j}\right)+\frac{\beta(y)}{2 \pi i} \int_{C_{2}} \frac{f(x, w) d w}{\beta(w)(w-y)}
$$

where $\beta(w)$ is defined as in (28) and $C_{2}$ is a simple closed contour in the region $D_{w}$ of analyticity of $f(x, w)$ (x fixed) and containing the points $y_{0}, \cdots, y_{m}$ in its interior.

Now assume that $f(z, w)$ is simultaneously analytic in $D_{z} \times D_{w}$. From (34) and (35) we obtain

$$
f(x, y)=H_{M, N}(x, y)+R(x, y),
$$

where $H_{M, N}(x, y)$ is the interpolation polynomial of Theorem 2 , and $R(x, y)$ is the error of interpolation. Simplifying the expression for the error, we obtain

$$
\begin{aligned}
R(x, y) & =\frac{\alpha(x)}{2 \pi i} \int_{C_{1}} \frac{f(z, y) d z}{\alpha(z)(z-x)}+\frac{\beta(y)}{2 \pi i} \int_{C_{2}} \frac{f(x, w) d w}{\beta(w)(w-y)} \\
- & \frac{\alpha(x) \beta(y)}{(2 \pi i)^{2}} \int_{C_{1}} \int_{C_{2}} \frac{f(z, w) d z d w}{\alpha(z) \beta(w)(z-x)(w-y)} .
\end{aligned}
$$

Again, with the help of Cauchy's integral formula for $f(x, w)$ and $f(z, y)$, we can write (37) as

$$
R(x, y)=(2 \pi i)^{-2} \int_{C_{1}} \int_{C_{2}} \frac{\alpha(x) \beta(w)+\alpha(z) \beta(y)-\alpha(x) \beta(y)}{\chi(z) \beta(w)(z-x)(w-y)} f(z, w) d z d w .
$$

\section{Acknowledgement}

We are grateful to Professor M. K. Jain for encouragement and helpful comments.

\section{References}

[1] A. Spitzbart, 'A generalization of Hermite's interpolation formula', Amer. Math. Monthly 67 (1960), 42-46.

[2] A. C. Ahlin, 'A bivariate generalization of Hermite's interpolation formula', Math. Comp. 18 (1964), 264-273.

[3] P. J. Davis, Interpolation and Approximation, (Blaisdell, New York, 1963).

Indian Institute of Technology

Hauz Khas

New Delhi-29, India. 